\title{
Bone marrow stem cells contribute to repair of the ischemically injured renal tubule
}

\author{
Sujata Kale, ${ }^{1}$ Anil Karihaloo, ${ }^{1}$ Paul R. Clark, ${ }^{2}$ Michael Kashgarian, ${ }^{3}$ Diane S. Krause, ${ }^{2}$ \\ and Lloyd G. Cantley ${ }^{1}$ \\ ${ }^{1}$ Section of Nephrology, \\ ${ }^{2}$ Department of Laboratory Medicine, and \\ ${ }^{3}$ Department of Pathology, Yale University School of Medicine, New Haven, Connecticut, USA
}

\begin{abstract}
The paradigm for recovery of the renal tubule from acute tubular necrosis is that surviving cells from the areas bordering the injury must migrate into the regions of tubular denudation and proliferate to re-establish the normal tubular epithelium. However, therapies aimed at stimulating these events have failed to alter the course of acute renal failure in human trials. In the present study, we demonstrate that $\mathrm{Lin}^{-} \mathrm{Sca}-1^{+}$cells from the adult mouse bone marrow are mobilized into the circulation by transient renal ischemia and home specifically to injured regions of the renal tubule. There they differentiate into renal tubular epithelial cells and appear to constitute the majority of the cells present in the previously necrotic tubules. Loss of stem cells following bone marrow ablation results in a greater rise in blood urea nitrogen after renal ischemia, while stem cell infusion after bone marrow ablation reverses this effect. Thus, therapies aimed at enhancing the mobilization, propagation, and/or delivery of bone marrow stem cells to the kidney hold potential as entirely new approaches for the treatment of acute tubular necrosis.
\end{abstract}

This article was published online in advance of the print edition. The date of publication is available

from the JCI website, http://www.jci.org. J. Clin. Invest. 112:42-49 (2003). doi:10.1172/JCI200317856.

\section{Introduction}

Acute tubular necrosis (ATN) of the kidney is the most common injury affecting renal tubular function and is responsible for the majority of cases of acute renal failure in the hospital setting (1). A major cause of ATN is ischemic renal injury following episodes of hypotension or surgical cross-clamping of the aorta and/or renal arteries. This form of renal injury has been extensively studied in animal models of renal artery crossclamping and has been shown to be due to necrosis of the S3 segment of the proximal tubule and, to a lesser degree, the thick ascending $\operatorname{limb}(2,3)$. Both of these tubular segments reside in the outer medulla of the kidney, where oxygen tension is low but transport requirements remain high, thus producing an environment where a fall in perfusion produces an imbalance in energy demand and supply (4).

Following ischemic injury, tubular cells have been found to undergo frank necrosis, apoptosis, detach-

Received for publication January 14, 2003, and accepted in revised form April 22, 2003.

Address correspondence to: Lloyd Cantley, Section of Nephrology, Yale University School of Medicine, 333 Cedar Street, LMP 2093, New Haven, Connecticut 06437, USA. Phone: (203) 785-7110; Fax: (203) 785-4904;

E-mail: lloyd.cantley@yale.edu.

Conflict of interest: The authors have declared that no conflict of interest exists.

Nonstandard abbreviations used: acute tubular necrosis (ATN); bone marrow-derived stem cell (BMSC); bone marrow transplantation (BMT); lineage positive $\left(\mathrm{Lin}^{+}\right)$; ischemia/reflow (I/R); blood urea nitrogen (BUN); stem cell transplant (SCT); bone marrow ablation (BMA). ment, or dedifferentiation into a more mesenchymal phenotype. The dedifferentiated cells appear to spread out and to migrate into regions of denuded basement membrane, and they eventually proliferate to repopulate and repair the injured tubule (1). These dedifferentiated cells are thus believed to represent a population of stem cells residing in the kidney (5). Multiple studies have investigated the role of growth factors such as HGF, EGF, and IGF-1 in enhancing the process of tubular repair in animals, although trials of growth factors in humans have yet to show benefit for the recovery from ATN.

During the past several years, a great deal of attention has been focused on the plasticity of bone marrow-derived stem cells (BMSCs). Traditionally, stem cells were believed to be lineage restricted and organ specific. However, recent studies have demonstrated that BMSCs from both mice and humans have the ability to cross lineage boundaries and to form functional components of other tissues, expressing tissuespecific proteins in organs such as heart, liver, brain, skeletal muscle, and vascular endothelium (6-11). The possibility that BMSCs might functionally contribute to the renal tubule has been more controversial. Three studies have demonstrated that when female kidneys were transplanted into male recipients, Y chromosome-bearing cells were found in the transplanted kidney (12-14). However, in two of the studies these cells represented only a small percentage of the total cells present, and in one study they were believed to represent interstitial lymphocytes rather than tubular epithelial cells. Using whole-bone marrow transplan- 
tation in the mouse, Poulsom and coworkers demonstrated that bone marrow-derived cells could populate the renal tubular epithelium (13). In a separate study, transplantation of a select population of BMSCs into lethally irradiated mice resulted in appearance of bone marrow-derived epithelial cells in the liver, gastrointestinal tract, and skin, but bone marrow-derived cells were not reported in the kidney (15).

A feature of several of the models in which transplanted bone marrow cells have been found to functionally reconstitute an organ has been ischemic injury of that organ $(11,16)$. We therefore used a mouse model of ischemic renal injury to determine whether BMSCs can contribute to the repopulation of injured renal tubules. In this study we demonstrate that BMSCs not only have the capacity to differentiate into renal epithelial cells in vivo, but that cells derived from BMSCs represent the majority of the cells that reconstitute the necrotic S3 segment of the renal tubule. These results suggest that approaches based on the mobilization and delivery to the kidney of the appropriate bone marrow cells should be investigated in our attempts to improve the outcome of patients with ATN.

\section{Methods}

Isolation and transplantation of bone marrow. Six- to eightweek-old Rosa26 mice (B6;129S-Gtrosa26; The Jackson Laboratory, Bar Harbor, Maine, USA) transgenically expressing the bacterial lac $Z$ gene (17) were used as the donors for bone marrow transplantation (BMT) in accordance with a protocol approved by the Yale Animal Resources Committee. Femurs and tibiae were collected and bone marrow cells harvested as previously described $(18,19)$. For whole BMT, cells were resuspended in sterile PBS and $1 \times 10^{6}$ cells were used for transplantation. For transplantation of $\mathrm{Lin}^{-} \mathrm{Sca}-1^{+} \mathrm{c}-\mathrm{kit}^{+}$ BMSCs, whole bone marrow was first depleted of lineage positive $\left(\mathrm{Lin}^{+}\right)$cells using magnetic columns (Miltenyi Biotec Inc., Auburn, California, USA) and a cocktail of biotinylated lineage-specific antibodies (antiCD4, -CD8, -CD11, -B220, -Gr1, and -Ter119; Pharmingen, San Diego, California, USA). Lin ${ }^{-}$cells were obtained and labeled using anti-Sca-1 FITC and anti-c-kit phycoerythrin (Pharmingen). Lin-Sca- $^{-}{ }^{+} \mathrm{c}-\mathrm{kit}^{+}$cells were then collected using a FACSVantage flow cytometer (Becton Dickinson Immunocytometry Systems, San Jose, California, USA). Then $5 \times 10^{3} \mathrm{Lin}^{-} \mathrm{Sca}-1^{+} \mathrm{c}-\mathrm{kit}^{+}$cells were injected into the recipient mice, with control animals receiving an equal number of $\mathrm{Lin}^{+}$cells eluted from the magnetic columns.

Six- to eight-week-old C57BL/6J mice (The Jackson Laboratory) were used as recipients for BMT studies. Mice were given a sublethal radiation dose of $3.5 \mathrm{~Gy}$ using a ${ }^{137} \mathrm{Cs}$ irradiator, then anesthetized using metofane, and cells were injected via the retroorbital sinus. The mice were then maintained under sterile conditions; at the specified time points, mice were sacrificed, and kidneys were collected and either frozen in OCT or fixed in formalin and embedded in paraffin. Five- to seven-micrometer sections were cut and stained for $\beta$-galactosidase and/or megalin (as described below).

Ischemia/reflow experiments. Mice were anesthetized with $100 \mathrm{mg} / \mathrm{kg}$ ketamine and $10 \mathrm{mg} / \mathrm{kg}$ xylazine injected intraperitoneally, and a midline incision was made. For unilateral ischemia/reflow (I/R), the left renal pedicle was clamped for the indicated time using a vascular clamp (Fine Science Tools Inc., Foster City, California, USA). The abdomen was covered with gauze moistened in PBS, and the mice were maintained at $37^{\circ} \mathrm{C}$ using a warming pad. At the specified time points, the clamp was removed and reperfusion was confirmed visually. To determine the extent of acute injury, control mice were sacrificed 24 hours after I/R, and kidneys were collected and processed for histology using $H \&$ E staining.

To induce acute renal failure in the mice, both renal pedicles were clamped for the indicated time, followed by reperfusion and closing of the abdominal wound. The mice received $500 \mu \mathrm{l}$ of PBS subcutaneously at the end of the procedure to prevent dehydration. For experiments involving stem cell infusion after I/R, cells were infused in $50 \mu \mathrm{l}$ PBS via the retroorbital sinus approximately 2.5 hours after reflow. A pilot experiment in control mice revealed that 25 minutes of bilateral renal I/R resulted in a modest rise in blood urea nitrogen (BUN) from $22.4 \mathrm{mg} / \mathrm{dl}$ to $27.4 \mathrm{mg} / \mathrm{dl}$ on day 4 , with return to base line by 1 week. Thirty minutes of bilateral I/R resulted in a peak BUN of $54 \mathrm{mg} / \mathrm{dl}$ on day 4 , and a slow return to base line by 3 weeks. Thirty-five minutes of bilateral I/R resulted in a peak BUN of $374 \mathrm{mg} / \mathrm{dl}$ with accompanying severe lethargy and anorexia. Therefore, 30 minutes of bilateral $\mathrm{I} / \mathrm{R}$ was chosen to examine reversible renal tubular ischemic injury. Blood samples for BUN values were obtained prior to renal ischemia and 2, 5, and 7 days after ischemia. BUN measurements were performed in the laboratory of Gerald Schulman (Yale University) using a protocol from Roche Diagnostics Systems Inc. (Somerville, New Jersey, USA).

Detection of $\beta$-galactosidase and megalin. Cells derived from the Rosa 26 mice were identified by enzymatic detection of bacterial $\beta$-galactosidase activity. Cryosections of the kidneys were fixed in $0.2 \%$ glutaraldehyde for 5 minutes at room temperature. Slides were washed three times with PBS ( $\mathrm{pH}$ 7.5). Sections were incubated at $37^{\circ} \mathrm{C}$ for $8-16$ hours with the $\mathrm{X}$-gal staining solution prepared in PBS. The staining solution contained 5 $\mathrm{mM} \mathrm{K}_{3} \mathrm{Fe}(\mathrm{CN})_{6}, 5 \mathrm{mM} \mathrm{K}_{4} \mathrm{Fe}(\mathrm{CN})_{6} \cdot 3 \mathrm{H}_{2} \mathrm{O}, 2 \mathrm{mM} \mathrm{MgCl}_{2}$, pH 7.5 (Sigma Chemical Co., St. Louis, Missouri, USA) and $1 \mathrm{mg} / \mathrm{ml} \mathrm{X-gal} \mathrm{(American} \mathrm{Bioanalytical,} \mathrm{Natick,}$ Massachusetts, USA). Sections were then washed with PBS and counterstained with neutral fast red solution (Vector Laboratories Inc., Burlingame, California, USA). The presence and percentage of blue tubules were determined microscopically at $\times 40$ by counting of a total of 200-250 tubules from four random fields for each kidney. For detection of megalin, sections were stained with X-gal as described above and then postfixed in methanol and rinsed in PBS. Sections were fur- 


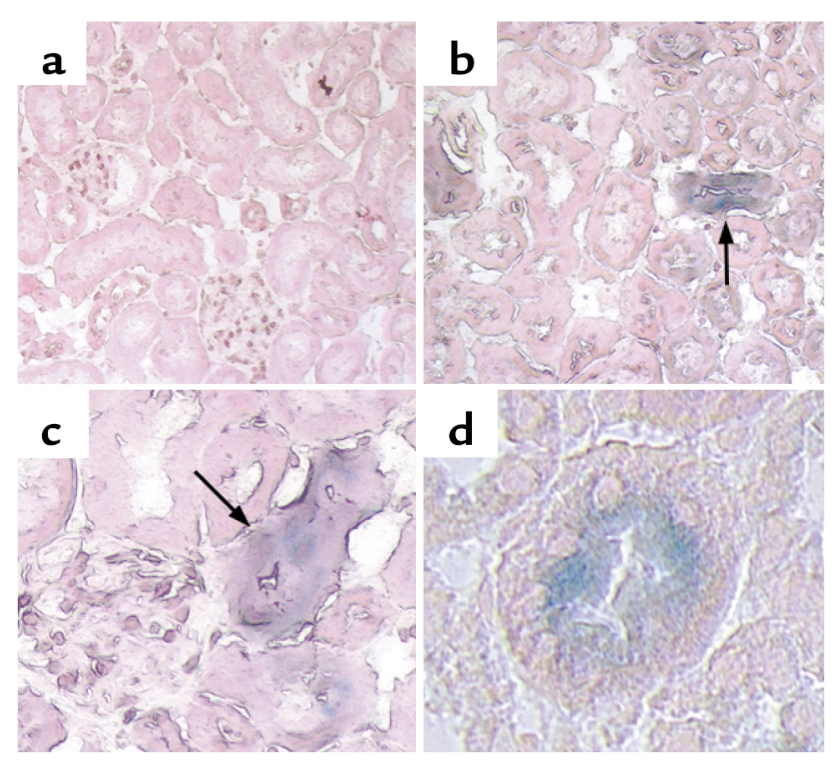

Figure 1

Bone marrow-derived cells populate the renal tubule. Transplantation of whole bone marrow from Rosa 26 mice into $C 57 \mathrm{BL} / 6 \mathrm{~J} \mathrm{recip-}$ ient mice results in the spontaneous appearance of $\beta$-galactosidase-positive cells (blue) in the tubules of the renal cortex of the transplanted mice after 12 weeks (arrows). Control mice (a) do not have $\beta$-galactosidase-positive tubules. $\mathbf{a}$ and $\mathbf{b}, \times 20 ; \mathbf{c}, \times 40 ; \mathbf{d}, \times 60$.

ther blocked for 30 minutes using $10 \%$ goat serum followed by incubation with rabbit anti-megalin (a kind gift of Dan Biemesderfer, Yale University School of Medicine, New Haven, Connecticut, USA) at 1:500 dilution for 1 hour. This was followed by Alexa fluor-conjugated goat anti-rabbit antibody (Molecular Probes Inc., Eugene, Oregon, USA).

Mobilization of bone marrow stem cells following ischemic injury. Twenty-four hours after 25 minutes of unilateral I/R or sham operation, mice were anesthetized with metofane, and peripheral blood was obtained via the retroorbital sinus using a heparinized capillary pipette (Fisher Scientific Co., Pittsburgh, Pennsylvania, USA). Red blood cells were lysed using PharM Lyse (Pharmingen), and remaining peripheral blood cells were labeled with biotin-conjugated lineage-specific antibodies (anti-CD4, -CD8, -CD11, -B220,-Gr1, and -Ter119) and anti-biotin phycoerythrin (Miltenyi Biotec Inc.) as well as FITC-conjugated anti-Sca-1 antibodies (Pharmingen). Flow cytometry was performed using a FACSVantage flow cytometer (Becton Dickinson Immunocytometry Systems) with corresponding isotype antibodies used as controls. A minimum of 10,000 cells were interrogated in each sample. Data were analyzed using CellQuest software (Becton Dickinson Immunocytometry Systems), and the percentage of $\mathrm{Lin}^{-} \mathrm{Sca}-1^{+}$cells was determined.

\section{Results}

Adult bone marrow contains cells capable of populating the renal tubule. Given the plasticity of adult BMSCs, we initially determined whether transplanted whole-bone marrow cells had the ability to home to the kidney tubular epithelium. Six C57BL/6J mice received sublethal irradiation followed by intravenous injection of either saline (control mice, $n=2$ ) or $1 \times 10^{6}$ wholebone marrow cells obtained from Rosa26 mice $(n=4)$. Rosa2 6 mice carry the lac $Z$ gene, making it possible to identify transplanted donor cells in the recipient mice by staining for $\beta$-galactosidase activity.

At 3 and 12 weeks after BMT, one control mouse and two transplanted mice were sacrificed, and the kidneys were sectioned and stained for $\beta$-galactosidase activity. Control mice that did not receive BMT had no $\beta$-galactosidase-positive cells in the kidneys at either 3 or 12 weeks (Figure 1a). In mice sacrificed 3 weeks after BMT from Rosa 26 donors, we also failed to detect $\beta$-galactosidase-positive cells in the kidneys. However, 12 weeks following BMT, $\beta$-galactosidase-positive cells were found in the kidneys and, at higher power, were found to be within the tubules themselves (Figure 1,b-d), with approximately $2-5 \%$ of the tubules containing bone marrow-derived cells. No $\beta$-galactosidase-positive cells were detected in the arteries or glomeruli in these experiments. These results demonstrate that transplanted whole bone marrow from adult mice contains cells that are capable of homing to the kidney and incorporating into the tubular epithelium. The paucity of $\beta$-galactosidase-positive tubules found is consistent with previous reports that used transplantation of female kidneys into male recipients and suggests that bone marrow-derived cells may play only a modest role in normal tubular epithelial turnover.

Ischemia induces mobilization of BMSCs and repopulation of the $S 3$ segment of the renal tubule. We next examined the possibility that adult stem cells are involved in repair-

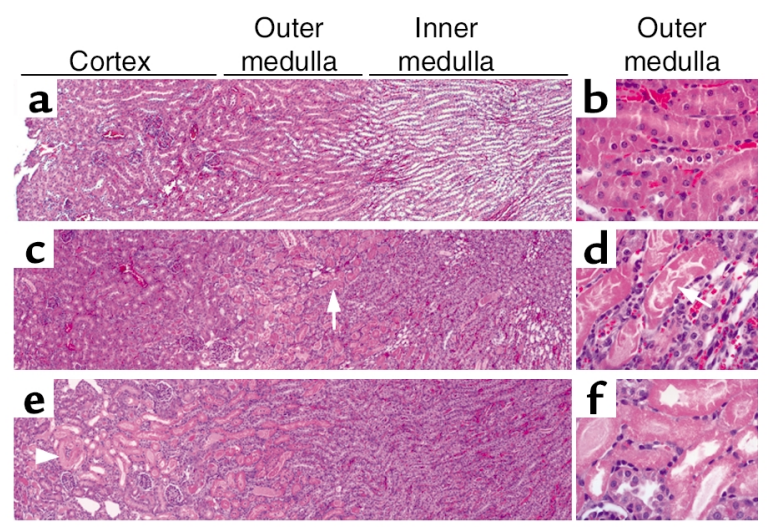

Figure 2

Mouse model of I/R. Low-magnification $(\times 4$; a, $\mathbf{c}$, and $\mathbf{e})$ and highmagnification $(\times 40 ; \mathbf{b}, \mathbf{d}$, and $\mathbf{f})$ views of $\mathrm{H} \& \mathrm{E}$-stained sections from control kidneys ( $\mathbf{a}$ and $\mathbf{b}$ ) and kidneys 24 hours after warm I/R for 25 minutes ( $\mathbf{c}$ and $\mathbf{d}$ ) and 30 minutes (e and $\mathbf{f}$ ). Twenty-five minutes of warm ischemia results in swelling of the cortical and inner medullary tubular cells and selective necrosis of the S3 segment of the proximal tubule in the outer medulla ( $\mathbf{c}$ and $\mathbf{d}$, arrows), whereas 30 minutes of warm ischemia causes more extensive necrosis of tubules in the outer medulla as well as necrosis of proximal tubules in the cortex (e, arrowhead; and $\mathbf{f}$ ). 

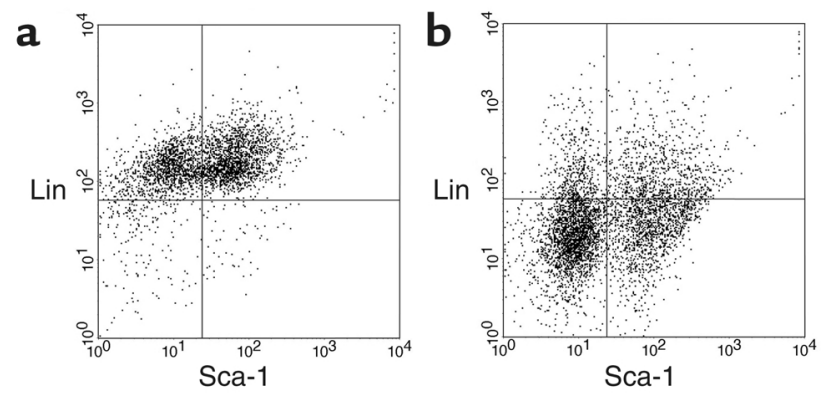

Figure 3

Ischemia mobilizes $\mathrm{Lin}^{-} \mathrm{Sca}-1^{+}$cells. Peripheral blood from a mouse 24 hours after sham operation (a) or 25 minutes of unilateral I/R (b) was labeled for detection of $\mathrm{Lin}^{+}$and Sca- $1^{+}$cells and analyzed on a FACS. Lin-Sca- ${ }^{+}$stem cells were not seen in the circulation of the control animals (a, lower right quadrant); the $1.4 \%$ of cells detected is equal to the value seen with the unrelated isotype control antibody and is therefore indistinguishable from background. In contrast, $23.8 \%$ of the circulating cells were $\mathrm{Lin}-\mathrm{Sca}-1^{+}$stem cells 24 hours after I/R (b, lower right quadrant).

ing the ischemically injured renal epithelium. For these studies we used the well-established model of I/R to examine regeneration of renal tubules following ATN. To determine the optimal clamping time for inducing selective injury to the S3 segment of the proximal tubule in the mouse, we clamped the left renal pedicle for periods of time that varied from 15 to 60 minutes and then unclamped it to restore flow; 24 hours later, we examined renal pathology. Ischemia times of 15-20 minutes induced swelling of the cells of the outer medulla but no frank necrosis. In agreement with published reports of this model (20), 25-27 minutes of ischemia resulted in patchy areas of necrosis of the proximal tubule cells in the outer medulla as compared with controls (Figure 2, a-d). Tubular segments of the thick ascending limb and collecting duct in the outer medulla were swollen, but not obviously necrotic. After 30 minutes of clamping, tubular necrosis in the outer medulla was less selective, and frank necrosis extended into the cortex (Figure 2, e and f).

\footnotetext{
Figure 4

Ischemia induces bone marrow cells to repopulate the tubules of the outer medulla. (a-f) Low-magnification $(\times 4 ; \mathbf{a}, \mathbf{c}$, and $\mathbf{e})$ and highmagnification ( $\times 40 ; \mathbf{b}, \mathbf{d}$, and $\mathbf{f})$ views of $X$-gal-stained kidney sections from mice that underwent 25 minutes of I/R 16 weeks after whole BMT, followed by a 1-week recovery period ( $\mathbf{a}$ and $\mathbf{b}$ ); mice 17 weeks after BMT that had not undergone renal ischemia ( $\mathbf{c}$ and $\mathbf{d}$ ); and mice that underwent 25 minutes of I/ $R$ with a 1 -week recovery period but did not undergo BMT (e and $\mathbf{f}$ ). I/R induced a marked increase in the total number of $\beta$-galactosidase-positive tubules, with the majority of those tubules confined to the region of necrosis in the outer medulla ( $\mathbf{a}$ and $\mathbf{b}$; blue tubules). ( $\mathbf{g}$ and $\mathbf{h}$ ) Transplantation of Lin-Sca- $1^{+}$c-kit ${ }^{+}$BMSCs followed 5 weeks later by 25 minutes of I/R resulted in a similar appearance of $\beta$-galactosidase-positive tubules in the outer medulla (h), while animals transplanted with lineagerestricted $\left(\mathrm{Lin}^{+}\right)$cells had no $\beta$-galactosidase-positive tubules after renal injury $(\mathbf{g})$. OM, outer medulla; IM, inner medulla.
}

To determine whether ischemic injury specifically induced the mobilization of bone marrow stem cells into the peripheral circulation, we subjected mice to 25 minutes of left renal ischemia or sham operation, followed 24 hours later by examination of the peripheral blood for the presence of Lin-Sca- $^{+}{ }^{+}$cells using flow cytometry. In the sham-operated mice, circulating Lin-Sca- $1^{+}$cells were not detectable above background (Figure 3a), whereas, after ischemic renal injury, these cells made up $20-24 \%$ of the non-rbc peripheral circulating cells (Figure $3 \mathrm{~b}$ ). Therefore, $\mathrm{Lin}^{-} \mathrm{Sca}-1^{+} \mathrm{c}-\mathrm{kit}^{+}$ BMSCs would be predicted to constitute $2-2.5 \%$ of the circulating cells after renal ischemia, as they typically make up approximately $10 \%$ of the $\mathrm{Lin}^{-} \mathrm{Sca}-1^{+}$fraction.

The ability of renal ischemia to mobilize Lin-Sca- $1^{+}$ cells from the bone marrow suggested that these cells might provide a substantial contribution to the repair of the necrotic renal tubules. To investigate this possibility, 11 mice were subjected to sublethal irradiation followed by whole BMT with $5 \times 10^{6}$ cells per mouse. Sixteen weeks after transplantation, six mice underwent clamping of the left renal pedicle for 25 minutes followed by reperfusion for 1 week, with the remaining five mice serving as sham-operated controls. Sections from the kidneys 7 days after surgery revealed a marked increase in the total number of $\beta$-galactosidase-positive cells in kidneys that had undergone $\mathrm{I} / \mathrm{R}$, with the majority of these cells being located in the tubules of

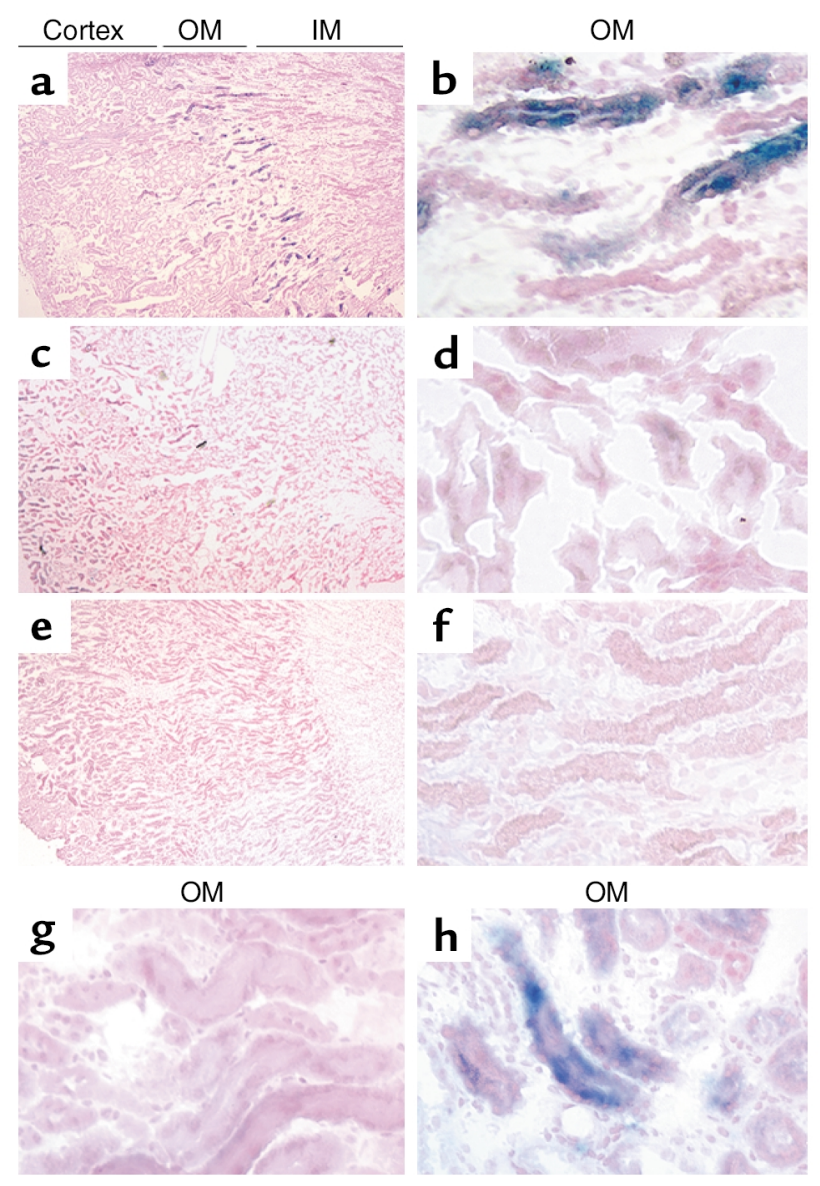


Table 1

Quantitation of $\beta$-galactosidase-positive tubules

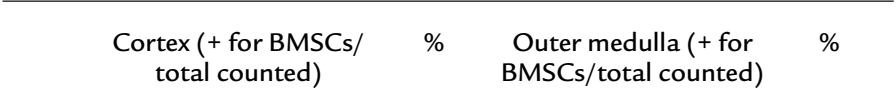

Ischemia/Reflow

$\begin{array}{ccccc}\text { Ischemic kidney } & 19 / 256 & 3.5 & 43 / 225 & 19.1 \\ & 7 / 247 & 2.8 & 40 / 225 & 17.8 \\ 14 / 242 & 5.7 & 55 / 225 & 24.4 \\ 5 / 231 & 2.2 & 41 / 233 & 17.6 \\ 15 / 217 & 6.9 & 54 / 198 & 27.3 \\ 11 / 196 & 5.6 & 39 / 203 & 19.2 \\ & & \text { Average }=4.5 \pm 0.8 & & \text { Average }=20.9 \pm 1.6 \\ \text { Contralateral kidney } & 11 / 213 & 5.2 & 9 / 255 & 3.5 \\ & 11 / 269 & 4.1 & 8 / 247 & 3.2 \\ & 12 / 239 & 5.0 & 6 / 230 & 2.6 \\ & 10 / 225 & 4.4 & 7 / 225 & 3.1 \\ & 10 / 200 & 5.0 & 6 / 200 & 3.0 \\ & 9 / 225 & 4.0 & 5 / 194 & 2.6 \\ \text { Control } & & \text { Average }=4.6 \pm 0.2 & & \text { Average }=3.0 \pm 0.1 \\ & & & & \\ & & 3.8 & 5 / 208 & 2.4 \\ & & 2.2 & 1 / 202 & 0.5 \\ & & & 4 / 251 & 1.6 \\ & 8 / 230 & 3.4 & 8 / 215 & 3.7 \\ & 12 / 209 & 5.7 & 9 / 225 & 4.0 \\ & 8 / 205 & 3.9 & & \text { Average }=2.4 \pm 0.7\end{array}$

Mice undergoing whole BMT were subjected 15 weeks later to unilateral I/R $(n=6)$ or sham operation (control, $n=5$ ), followed by 1 week of recovery and quantitation of the number of $\beta$-galactosidase-positive tubules in the cortex and outer medulla of kidney sections. For each animal, between 194 and 269 tubules were counted from four random fields and scored for the presence or absence of blue cells. Data are presented as \pm SEM.

the outer medulla (Figure 4, a and b). The contralateral kidney, and kidneys from the mice that had not undergone renal ischemia, again revealed only a small number of $\beta$-galactosidase-positive cells, distributed primarily in the renal cortex (Figure 4, $c$ and d). A second group of control mice that underwent $\mathrm{I} / \mathrm{R}$ in the absence of prior BMT showed no $\beta$-galactosidase-positive tubules (Figure 4, e and f).

Quantitation of the number of $\beta$-galactosidase-positive tubules from these experiments revealed that ischemia induced an increase in $\beta$-galactosidase-positive tubules from $2.4 \%$ to $20.9 \%(P<0.001)$ in the outer medulla of ischemically injured kidneys but did not alter the number of tubules with detectable bone marrow-derived cells in the cortex (Table 1). Furthermore, there was no increase in $\beta$-galactosidase-positive tubules in the contralateral kidney, and there were no $\beta$-galactosidase-positive tubules detected in the inner medulla of any animal. Bone marrow cytopreparations revealed that $24.5 \% \pm 6 \%$ of the bone marrow cells were $\beta$-galactosidase-positive 17 weeks after BMT. A separate experiment in which mice underwent left renal I/R 2 weeks after BMT, followed by 1 week of recovery, demonstrated that the number of $\beta$-galactosidase-positive tubules in the outer medulla increased from $0 \%$ to $8.5 \%$ following renal ischemia, with bone marrow cytopreparations confirming that $3.3 \%$ of the bone marrow cells were derived from the Rosa 26 donor at this early time after transplantation.

To determine whether the cells capable of regenerating the renal epithelium reside in the traditional bone marrow stem cell fraction, we repeated the $\mathrm{I} / \mathrm{R}$ experiment in mice transplanted with either $\mathrm{Lin}^{-} \mathrm{Sca}-1^{+} \mathrm{c}-\mathrm{kit}^{+}$cells (stem cell transplant [SCT] group, $n=3$ ) or $\mathrm{Lin}^{+}$cells (control group, $n=3$ ). The left kidneys of mice in the two groups were subjected to 25 minutes of surgical I/R 5 weeks after stem cell transplantation, as before. X-gal staining of the kidney sections 1 week later revealed that $\beta$-galactosidase-positive tubules constituted $0 \%$ of the outer medulla in mice that received $\mathrm{Lin}^{+}$cells, but approximately $22 \%$ of the outer medulla in mice that received $\mathrm{Lin}-\mathrm{Sca}-1^{+} \mathrm{c}-\mathrm{kit}{ }^{+}$BMSCs (Figure 4, g and h). Bone marrow cytopreparations demonstrated that, in the animals that received $\mathrm{Lin}^{-} \mathrm{Sca}-1^{+} \mathrm{c}-\mathrm{kit}^{+}$cells, $22.9 \% \pm 6.2 \%$ of the bone marrow cells were derived
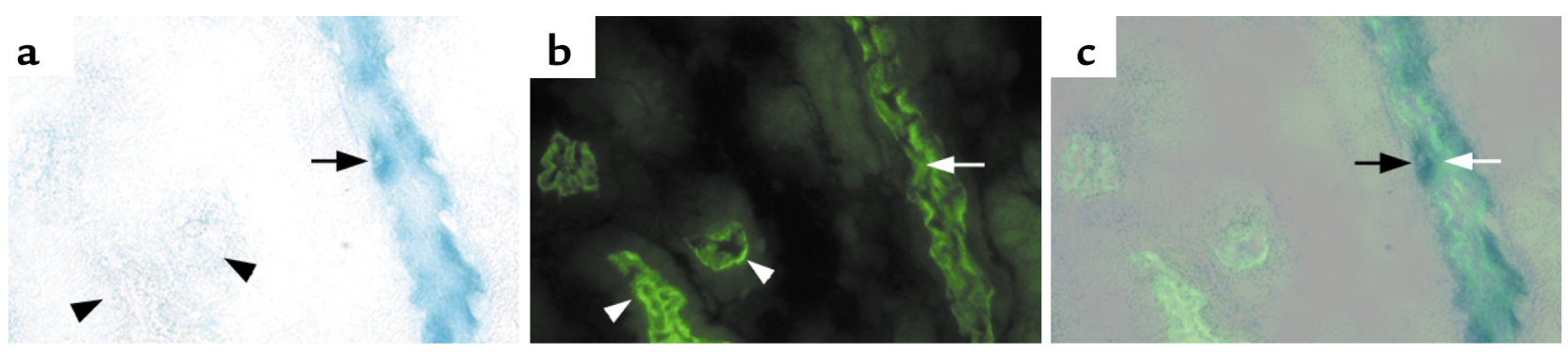

\section{Figure 5}

Bone marrow-derived cells express the renal proximal tubule-specific marker megalin. Sections from kidneys of mice that underwent 25 minutes of ischemia 16 weeks after BMT, followed by 1 week of reflow, were double stained with X-gal (a) and the proximal tubule-specific marker megalin (b). Tubules from the outer medulla that contain cells derived from the bone marrow of the Rosa26 mouse (a, arrow) express megalin on their apical surface (b, arrow), with the merged image showing double staining of the same cell (c, arrows). Two other S3 tubules are shown that express megalin (b, arrowheads) but are not $\beta$-galactosidase positive (a, arrowheads). Megalin and $\beta$-galactosidase-negative tubules seen faintly in the background represent medullary thick ascending limbs and collecting ducts. 
from the Rosa26 mouse donor, whereas none of the $\mathrm{Lin}^{+}$cells had engrafted. Thus it is specifically the $\mathrm{Lin}^{-} \mathrm{Sca}-1^{+} \mathrm{c}-\mathrm{kit}^{+}$fraction of the bone marrow that contains cells capable of populating the renal tubule following ischemic injury.

In order to determine whether the bone marrowderived cells found in the regenerated proximal tubule had differentiated into renal tubular epithelial cells, we performed $\beta$-galactosidase assays followed by staining of the same section for the proximal tubule-specific marker megalin (21). Examination of the outer medulla 1 week after 25 minutes of ischemia revealed that approximately $20 \%$ of the proximal tubules contained $\beta$-galactosidase-positive cells (Figure 5a), with immunohistochemistry revealing that these bone marrow-derived cells express megalin in the typical apical location (Figure $5 \mathrm{~b}$ ). Overlay of these images revealed that the same cells contained $\beta$-galactosidase in their cytoplasm and megalin on their apical surface (Figure 5c). Staining of kidneys from transplanted animals that did not undergo renal I/R demonstrated that the bone marrow-derived cells in these kidneys coexpressed megalin as well, suggesting that the majority of these cells have differentiated into a proximal tubule phenotype (data not shown).

Bone marrow stem cells contribute to functional repair of the ischemically injured tubule. To test our hypothesis that bone marrow stem cells functionally contribute to renal tubule repair, 14 mice were divided into two experimental groups: bone marrow ablation (BMA, $n=7$ ), and BMA followed by stem cell transplantation (BMA-SCT, $n=7$ ). The mice were subjected to BMA by lethal irradiation (11 Gy/animal) followed 12 hours later by bilateral renal pedicle cross-clamping for 30 minutes. Approximately 2.5 hours after reperfusion, seven animals were injected either with $2.9 \times 10^{5} \mathrm{Lin}^{-}$ bone marrow cells resuspended in $50 \mu$ l of PBS (BMASCT group), or with $50 \mu \mathrm{l}$ PBS vehicle (BMA group). Three animals in each group died within 48 hours of surgery due to postoperative infection and were excluded from the analysis. The remaining eight animals showed no signs of infection and continued to eat and drink for the remainder of the experimental period, although their food intake was judged to be somewhat decreased. A third group of mice underwent $30 \mathrm{~min}$ utes of bilateral renal ischemia in the absence of lethal irradiation (control, $n=4$ ). Since the BMA-SCT group was predicted to engraft the transplanted cells during the second week, whereas the BMA group was expected to begin dying during this time, the experiment was terminated on day 7 to minimize the potential effects of improvement of red blood cell, white blood cell, and platelet counts on renal recovery. Peripheral blood counts on day 7 reflected the lethal irradiation but were not significantly different between the two experimental groups (average white blood cell count $=1,000 / \mu \mathrm{l}$ in both BMA and BMA-SCT [normal murine white blood cell count $=5,000-8,000 / \mu \mathrm{l}]$; average hematocrit $=16.5 \%$ in BMA and 16.3\% in BMA-SCT [normal murine hema- tocrit $=50 \%$; average platelet count $=80,000 / \mu \mathrm{l}$ in BMA and $110,000 / \mu 1$ in BMA-SCT [normal murine platelet count $>700,000 / \mu 1])$.

Animals undergoing BMA followed by $\mathrm{I} / \mathrm{R}$ and stem cell transplantation demonstrated a peak BUN of $48.0 \pm 12.9$ $\mathrm{mg} / \mathrm{dl}$ on day 2 after I/R with a subsequent decline to $40.6 \pm 9 \mathrm{mg} / \mathrm{dl}$ by day 7 , a pattern that was indistinguishable from that in control mice (Figure 6a). In contrast, animals that did not receive stem cells had a peak BUN of $77.5 \pm 25 \mathrm{mg} / \mathrm{dl}$ on day 2 with no recovery during the 7-day experimental period (day $7 \mathrm{BUN}=75 \pm 1.8 \mathrm{mg} / \mathrm{dl}$ ). Of note, animals subjected to $11 \mathrm{~Gy}$ in the absence of renal
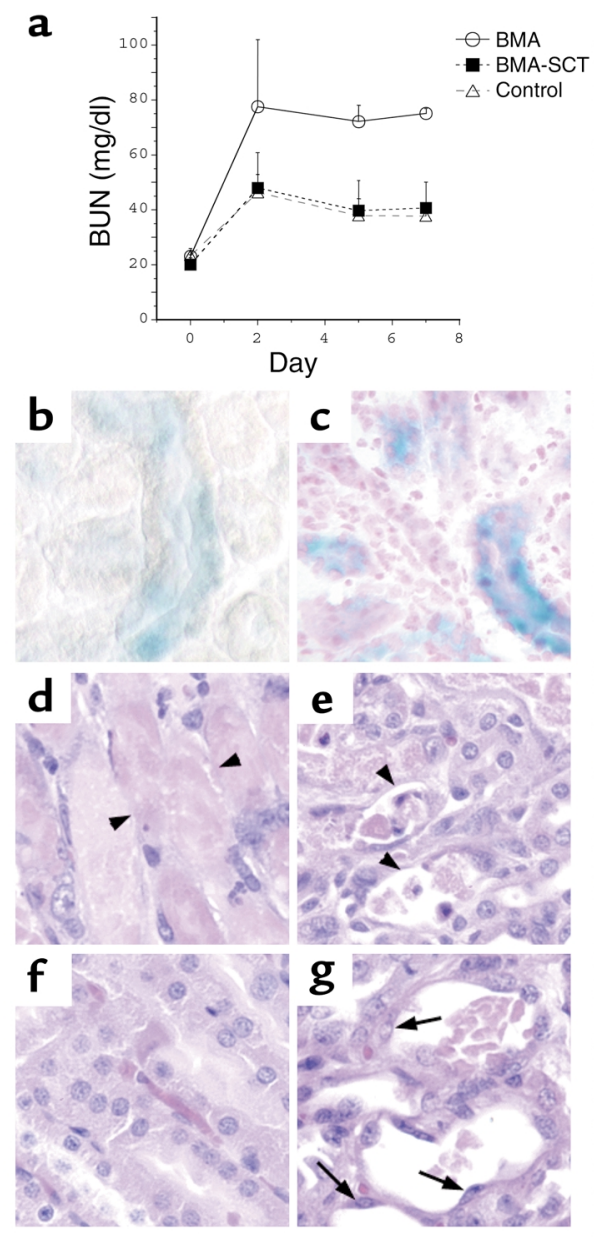

\section{Figure 6}

BMA worsens the course of ischemic ATN. (a) BUN values from mice that underwent bilateral ischemia for 30 minutes followed by 7 days of recovery. Mice underwent BMA prior to I/R (BMA); underwent BMA prior to $\mathrm{I} / \mathrm{R}$ and stem cell transplantation after $\mathrm{I} / \mathrm{R}$ (BMA-SCT); or underwent $\mathrm{I} / \mathrm{R}$ without $\mathrm{BMA}$ (control). ( $\mathbf{b}$ and $\mathbf{c}$ ) X-gal staining of kidney sections reveals multiple $\beta$-galactosidase-positive tubules in the kidneys of BMA-SCT animals 48 hours after I/R (b) and 7 days after $\mathrm{I} / \mathrm{R}(\mathbf{c})$. Of note, the sections in $\mathbf{b}$ were not counterstained with neutral fast red. (d-g) H\&E staining reveals tubular damage in the outer medulla of both groups, with extensive denudation of some of the tubular basement membranes in animals that underwent BMA alone ( $\mathbf{d}$ and $\mathbf{e}$, arrowheads). The majority of damaged tubules in the BMA-SCT group contain flattened cells lining the basement membrane ( $\mathbf{g}$, arrows), while other areas demonstrate almost complete resolution of the injury $(\mathbf{f}) . \times 40$. 
artery cross-clamping had no change in BUN (preirradiation $\mathrm{BUN}=17.2 \pm 0.6 \mathrm{mg} / \mathrm{dl}$, BUN 4 days after irradiation $=18.2 \pm 2.9 \mathrm{mg} / \mathrm{dl}, n=10$ ).

The observation that stem cell infusion limited the initial rise in BUN suggested that these cells must have reached the renal tubule within 48 hours of I/R. To examine this possibility, an additional group of three mice underwent BMA and stem cell infusion as described. These mice were sacrificed 48 hours after reperfusion, and kidney sections were examined for the presence of $\beta$-galactosidase-positive cells. At this early time point, multiple tubules were found to contain cells derived from the transplanted bone marrow (Figure 6b). Staining on day 7 revealed persistence of these cells in the outer medulla of animals in the BMA-SCT group (Figure 6c).

Renal histology on day 7 revealed a spectrum of injury in both groups. Kidneys from animals in the BMA group had some areas of extensive necrosis remaining in the outer medulla (Figure 6d), whereas other regions of the outer medulla revealed only patchy areas of necrosis (Figure 6e). Multiple tubules demonstrated persistent areas of basement membrane denudation (arrowheads), while some tubules had flattened cells lining these injured segments. In contrast, animals receiving stem cell transplants after BMA and I/R had large areas in which there was substantial resolution of the necrotic injury (Figure 6f) but also had significant regions in which tubular injury was still evident (Figure $6 \mathrm{~g})$. However, the tubules that did show persistent injury were judged to have less frank denudation and more cells lining the tubular basement membrane than were seen in the BMA control group (Figure $6 \mathrm{~g}$, arrows).

\section{Discussion}

Our results show that bone marrow stem cells have the ability to repair ischemic renal tubular injury, similar to what has been recently shown with ischemic injury to the myocardium and with retinal injury $(16,22,23)$. We demonstrate that 1 week after ischemia/reperfusion, the previously necrotic S3 segments of the proximal tubules are lined by cells of donor origin that have differentiated into renal epithelial cells. To achieve this, BMSCs have presumably migrated out of the bone marrow and then homed specifically to the injured area. This suggests that the injured kidney secretes a specific factor or factors that act on the bone marrow, causing the release of a large number of BMSCs into the circulation. The classic BMSCs are $\mathrm{Lin}^{-} \mathrm{Sca}-1^{+} \mathrm{c}-\mathrm{kit}^{+}$ cells that represent approximately $0.01 \%$ of the bone marrow cells but that are not found in detectable numbers in the peripheral blood $(24,25)$. However, these stem cells can be mobilized into peripheral blood by treatment with several growth factors, including steel factor and GM-CSF (26). In the present study, renal ischemia dramatically mobilized BMSCs into the circulation, apparently facilitating the repair process.

In our animals, sublethal irradiation followed by transplantation of $5 \times 10^{6}$ whole-bone marrow cells per mouse resulted in progressive engraftment of those cells into the recipient bone marrow. After 3 weeks, approximately $3 \%$ of the bone marrow cells were donor-derived, while after 17 weeks, approximately $25 \%$ of the bone marrow cells had come from the donor. Thus, considering that the majority of the BMSCs in these mice were not from the Rosa 26 donor, our finding that approximately $20 \%$ of the tubules in the outer medulla of ischemic kidneys contained segments of $\beta$-galactosidase-positive cells suggests that the majority of the cells that repopulate the necrotic tubule come from the bone marrow.

Our results show that BMA worsened the initial rise in BUN following bilateral renal ischemia and also slowed the histologic recovery of the tubules in the outer medulla. The fact that stem cell infusion prevented both of these effects suggests that stem cells play an early role in limiting the initial injury as well as a late role in enhancing tubular repair. While it seems unlikely that stem cells have fully differentiated into a functional tubular epithelial phenotype within 48 hours, their presence in the tubules supports the alternative possibility that the initial role of these cells is to limit tubular backleak of the glomerular filtrate, thus maintaining GFR $(27,28)$. It is important to note that if there are stem cells residing within the renal parenchyma, lethal irradiation could potentially slow repair of the tubules by these cells, in addition to eliminating repair by the bone marrow stem cells.

These findings suggest that repair of the renal tubule may occur as a cooperative effort involving both the local actions of surviving tubular cells within the injured tubular segments, and the mobilization and homing of bone marrow stem cells to the areas of injury. These BMSCs appear to differentiate into epithelial cells appropriate to the tubular segment that they populate and to provide a functional role in limiting the degree of acute renal failure. The present results provide the conceptual basis for the development of therapeutic strategies aimed at stimulating the proliferation, mobilization, and targeting of these stem cells in an effort to enhance recovery from acute renal failure.

\section{Acknowledgments}

The authors would like to thank Rocco Carbone (Yale Cancer Center Flow Cytometry Shared Resources) for help with flow cytometric analysis and flow sorting, Sue Ann Mentone, Steve Bennett, and Russell Mortimer for help in preparing tissue sections, and the staff of the Veterinary Clinical Services at Yale, especially Julia Merk and Krista Fowles, for assistance with the mouse surgeries. We also acknowledge NIH grant U24 DK-59635 and the technical assistance of Rebecca LePine and Gary Cline for the BUN measurements. This work was supported by NIH grants to L.G. Cantley and A. Karihaloo.

1. Thadhani, R., Pascual, M., and Bonventre, J.V. 1996. Acute renal failure. N. Engl. J. Med. 334:1448-1460.

2. Shanley, P.F., et al. 1986. Topography of focal proximal tubular necrosis after ischemia with reflow in the rat kidney. Am. J. Pathol. 122:462-468.

3. Witzgall, R., Brown, D., Schwarz, C., and Bonventre, J.V. 1994. Localiza- 
tion of proliferating cell nuclear antigen, vimentin, c-Fos, and clusterin in the postischemic kidney. Evidence for a heterogenous genetic response among nephron segments, and a large pool of mitotically active and dedifferentiated cells. J. Clin. Invest. 93:2175-2188.

4. van Why, S.K., et al. 1999. Thresholds for cellular disruption and activation of the stress response in renal epithelia. Am. J. Physiol. 277:F227-F234.

5. Al-Awqati, Q., and Oliver, J.A. 2002. Stem cells in the kidney. Kidney Int. 61:387-395.

6. Eglitis, M.A., and Mezey, E. 1997. Hematopoietic cells differentiate into both microglia and macroglia in the brains of adult mice. Proc. Natl. Acad. Sci. U. S. A. 94:4080-4085.

7. Theise, N.D., et al. 2000. Liver from bone marrow in humans. Hepatology. 32:11-16.

8. Krause, D.S. 2002. Plasticity of marrow-derived stem cells. Gene Ther. 9:754-758.

9. Ferrari, G., et al. 1998. Muscle regeneration by bone marrow-derived myogenic progenitors. Science. 279:1528-1530.

10. Hess, D.C., et al. 2002. Bone marrow as a source of endothelial cells and NeuN-expressing cells after stroke. Stroke. 33:1362-1368.

11. Orlic, D., et al. 2001. Mobilized bone marrow cells repair the infarcted heart, improving function and survival. Proc. Natl. Acad. Sci. U. S. A. 98:10344-10349.

12. Gupta, S., Verfaillie, C., Chmielewski, D., Kim, Y., and Rosenberg, M.E. 2002. A role for extrarenal cells in the regeneration following acute renal failure. Kidney Int. 62:1285-1290.

13. Poulsom, R., et al. 2001. Bone marrow contributes to renal parenchymal turnover and regeneration. J. Pathol. 195:229-235.

14. Grimm, P.C., et al. 2001. Neointimal and tubulointerstitial infiltration by recipient mesenchymal cells in chronic renal-allograft rejection. N. Engl. J. Med. 345:93-97.

15. Krause, D.S., et al. 2001. Multi-organ, multi-lineage engraftment by a single bone marrow-derived stem cell. Cell. 105:369-377.

16. Otani, A., et al. 2002. Bone marrow-derived stem cells target retinal astro- cytes and can promote or inhibit retinal angiogenesis. Nat. Med. 8:1004-1010

17. Zambrowicz, B.P., et al. 1997. Disruption of overlapping transcripts in the ROSA beta geo 26 gene trap strain leads to widespread expression of beta-galactosidase in mouse embryos and hematopoietic cells. Proc. Natl. Acad. Sci. U. S. A. 94:3789-3794.

18. Kale, S.A., and Rao, S.G. 1992. Haemopoietic stem cells during development of mouse embryo. Indian J. Exp. Biol. 30:371-376.

19. Kale, S.A., and Rao, S.G. 1992. Haematopoietic microenvironmental status of the 13-day mouse embryonal liver. Nowv. Rev. Fr. Hematol. 34:449-454.

20. Kelly, K.J., et al. 1996. Intercellular adhesion molecule-1-deficient mice are protected against ischemic renal injury. J. Clin. Invest. 97:1056-1063.

21. Biemesderfer, D., Nagy, T., DeGray, B., and Aronson, P.S. 1999. Specific association of megalin and the $\mathrm{Na}+/ \mathrm{H}+$ exchanger isoform NHE3 in the proximal tubule. J. Biol. Chem. 274:17518-17524.

22. Orlic, D. 2002. Stem cell repair in ischemic heart disease: an experimental model. Int. J. Hematol. 76(Suppl. 1):144-145.

23. Nakatomi, H., et al. 2002. Regeneration of hippocampal pyramidal neurons after ischemic brain injury by recruitment of endogenous neural progenitors. Cell. 110:429-441.

24. Spangrude, G.J., Heimfeld, S., and Weissman, I.L. 1988. Purification and characterization of mouse hematopoietic stem cells. Science. 241:58-62.

25. Uchida, N., Aguila, H.L., Fleming, W.H., Jerabek, L., and Weissman, I.L. 1994. Rapid and sustained hematopoietic recovery in lethally irradiated mice transplanted with purified Thy-1.1lo Lin-Sca-1+ hematopoietic stem cells. Blood. 83:3758-3779.

26. Fu, S., and Liesveld, J. 2000. Mobilization of hematopoietic stem cells. Blood Rev. 14:205-218.

27. Moran, S.M., and Myers, B.D. 1985. Pathophysiology of protracted acute renal failure in man. J. Clin. Invest. 76:1440-1448.

28. Myers, B.D., and Moran, S.M. 1986. Hemodynamically mediated acute renal failure. N. Engl. J. Med. 314:97-105. 